\title{
Impaired activity of circulating EPCs and endothelial function are associated with increased Syntax score in patients with coronary artery disease
}

\author{
BIN ZHANG ${ }^{1,2^{*}}$, DONG LI $^{2,3^{*}}$, GEXIU LIU $^{4-6}$, WENFENG TAN $^{1,2}$, GAOXING ZHANG $^{1,2}$ and JINLI LIAO ${ }^{7}$ \\ ${ }^{1}$ Department of Cardiovascular Disease, ${ }^{2}$ Clinical Experimental Center, ${ }^{3}$ Department of Intensive Care Unit, \\ Jiangmen Central Hospital, Affiliated Jiangmen Hospital of Sun Yat-Sen University, Jiangmen, Guangdong 529030; \\ ${ }^{4}$ Institute of Hematology, Medical College, ${ }^{5}$ Key Laboratory for Regenerative Medicine of The Ministry of Education, \\ ${ }^{6}$ Joint Laboratory for Regenerative Medicine, Jinan University, Guangzhou, Guangdong 510632; ${ }^{7}$ Division of Emergency \\ Medicine, Department of General Internal Medicine, Department of Emergency Intensive Care Unit, The First Affiliated Hospital, \\ Sun Yat-Sen University, Guangzhou, Guangdong 510080, P.R. China
}

Received February 24, 2020; Accepted February 10, 2021

DOI: $10.3892 / \mathrm{mmr} .2021 .11960$

\begin{abstract}
It has previously been shown that the number of endothelial progenitor cells (EPCs) is negatively correlated with Syntax score in patients with coronary artery disease (CAD). However, the association between alterations in EPC function and Syntax score is still unknown. The present study evaluated the association between the activity of EPCs as well as endothelial function and Syntax score in patients with CAD and investigated the underlying mechanisms. A total of 60 patients with CAD were enrolled in 3 groups according to Syntax score, and 20 healthy subjects were recruited as the control group. The number and migratory, proliferative and adhesive activities of circulating EPCs were studied. The endothelial function was measured by flow-mediated dilatation (FMD) and the levels of nitric oxide (NO) in plasma or secreted by EPCs were detected. The number and activity of circulating EPCs were lower in patients with a high Syntax score, which was similar to the alteration in FMD. The level of NO in plasma or secreted by EPCs also decreased as Syntax
\end{abstract}

Correspondence to: Dr Jinli Liao, Division of Emergency Medicine, Department of General Internal Medicine, Department of Emergency Intensive Care Unit, The First Affiliated Hospital, Sun Yat-Sen University, 58 Zhongshan Second Road, Yuexiu, Guangzhou, Guangdong 510080, P.R. China

E-mail: liaojli5@mail.sysu.edu.cn

Dr Gaoxing Zhang, Clinical Experimental Center, Jiangmen Central Hospital, Affiliated Jiangmen Hospital of Sun Yat-Sen University, 23 Haibang Street, Pengjiang, Jiangmen, Guangdong 529030, P.R. China

E-mail: zhanggaoxing11@sohu.com

*Contributed equally

Key words: coronary artery disease, Syntax score, endothelial progenitor cell, endothelial function, nitric oxide score increased. There was a negative association between FMD or circulating EPCs and Syntax score. A similar association was observed between the levels of NO in plasma or secreted by EPCs and Syntax score. Patients with CAD who had a higher Syntax score exhibited lower EPC numbers or activity and weaker endothelial function, which may be associated with attenuated NO production. These findings provide novel surrogate parameters for evaluation of the severity and complexity of CAD.

\section{Introduction}

Coronary artery disease (CAD) is the most common clinical manifestation of ischaemic heart disease and a leading cause of mortality worldwide $(1,2)$. A characteristic feature of CAD is the formation of atherosclerotic plaques, leading to stenosis or obstruction of the coronary artery. Endothelial dysfunction has been implicated as the initiating factor and key event in the pathogenesis of atherosclerosis (3). Therefore, restoration of endothelial activity may prevent the progression of CAD and decrease cardiovascular events.

Circulating endothelial progenitor cells (EPCs) promote neovascularization, repair endothelial injury and improve endothelial function $(4,5)$. Circulating EPCs are impaired in patients with CAD (5) and studies have reported an inverse correlation between the number of circulating EPCs and Syntax score $(6,7)$. Derived from coronary anatomy and lesion characteristics, the Syntax score was devised to grade the anatomical complexity and severity of coronary lesions in patients with CAD (8-11). Moreover, it has been reported as an independent predictor of long-term major adverse cardiac and cerebrovascular events in patients treated with percutaneous coronary intervention $(8,12,13)$. However, the association between the activity of EPCs, endothelial function and Syntax score in patients with CAD is still not clear.

Nitric oxide (NO) serves an important role in the regulation of EPC function and can promote the mobilization, proliferation and migratory activities of circulating EPCs (14-16). Previous studies have shown that the level of NO in plasma or 
secreted by EPCs were correlated to the activity of circulating EPCs and FMD $(17,18)$. Based on the aforementioned studies, it was hypothesized that circulating EPC and endothelial function may be negatively correlated with Syntax score in patients with CAD and may be associated with decreased NO production. Therefore, the present study detected the number and function of circulating EPCs, measured endothelial function and evaluated levels of NO in plasma or culture medium in patients with different Syntax scores. Moreover, the present study detected the correlation between flow-mediated dilatation (FMD) or circulating EPCs and Syntax score.

\section{Materials and methods}

Characteristics of patients. A total of 60 patients with CAD were enrolled in 3 groups according to syntax score: Low-(Syntax score $<22$ points; $n=20 ; 10$ male patients and 10 female patients; age, 45-75 years), middle-(Syntax score, 22-32 points; $n=20 ; 10$ male patients and 10 female patients; age, $45-75$ years) and high-risk (Syntax score $>32$ points; $n=20$; 10 male patients and 10 female patients; age, $45-75$ years) and 20 healthy subjects (10 male patients and 10 female patients; age, 45-75 years) were recruited as a control group. Patients and healthy subjected were recruited between January 2018 and January 2019. In addition, according to the number of lesioned coronary arteries (anterior descending branch, circumflex branch and right coronary artery), the patients were divided into single, double and triple lesion vessel groups. The patients with CAD were diagnosed by coronary angiography because of stable angina pectoris admitted to our hospital (Jiangmen Central Hospital). In consideration of the factors that influence the number of EPCs, patients were excluded on the basis of smoking, chronic renal insufficiency (serum creatinine $\geq 1.4 \mathrm{mmol} / \mathrm{l}$ ), abnormal liver function, infection, inflammation, diabetes, myocardial infarction, elevated troponin-I or creatine kinase-MB levels and malignant or autoimmune disease. Patients taking antiplatelet, anti-inflammatory, or hypolipidaemic agents were also excluded. Blood samples $(50 \mathrm{ml})$ were taken for routine biochemistry characteristics measurements and EPCs were isolated. Written informed consent was obtained from all patients and healthy subjects and the protocol conformed to the ethical guidelines of the 1975 Declaration of Helsinki and was approved by the Jiangmen Central Hospital Ethics Committee on Research on Humans.

Isolation of circulating EPCs. Blood samples $(35 \mathrm{ml})$ were diluted with sterile PBS. A total of $15 \mathrm{ml}$ human lymphocyte separation solution (cat. no. HY2015; Tianjin Haoyang Biological Products Technology Co., Ltd.) was added into a 50-ml centrifuge tube (cat. no. 430829; Corning, Inc.). A total of $25 \mathrm{ml}$ diluted peripheral blood was added along the tube wall before centrifugation at $811.7 \mathrm{x} \mathrm{g}$ at $4^{\circ} \mathrm{C}$ for $25 \mathrm{~min}$. A capillary pipette was used to transfer the clouded mononuclear cell layer into another sterile $50-\mathrm{ml}$ centrifuge tube. Then, $5 \mathrm{ml}$ erythrocyte lysate (cat. no. C3702-120 ml; Beyotime Institute of Biotechnology) was added and left to stand for $10 \mathrm{~min}$ at $4^{\circ} \mathrm{C}$. The mixture was diluted with pre-cooled PBS buffer and mixed before centrifugation at $377.3 \mathrm{x} \mathrm{g}$ at $4^{\circ} \mathrm{C}$ for $10 \mathrm{~min}$. The upper layer was removed using a capillary pipette and discarded. Following the addition of $20 \mathrm{ml}$ pre-cooled PBS buffer to the original centrifuge tube, the mixture was centrifuged again at $377.3 \mathrm{xg}$ at $4^{\circ} \mathrm{C}$ for $10 \mathrm{~min}$ and a capillary pipette was used to remove the upper layer. Then, $10 \mathrm{ml}$ pre-cooled PBS was added to the original centrifuge tube and mixed well. The mixture was transferred to another sterile $50-\mathrm{ml}$ centrifuge tube before centrifugation at $4^{\circ} \mathrm{C}$ at $377.3 \mathrm{x}$ g for $10 \mathrm{~min}$, and the upper layer was discarded. Isolated circulating EPCs were then cultured in EGM-2 (cat. no. CC-3162; Beijing Bitab Biotech Co., Ltd.) containing 20\% high-quality fetal bovine serum at $37^{\circ} \mathrm{C}$ for 7 days, $100 \mathrm{U} / \mathrm{ml}$ penicillin/streptomycin and $50 \mathrm{ng} / \mathrm{ml}$ VEGF $50 \mathrm{ng} / \mathrm{ml}$.

Identification and evaluation of circulating EPCs. Circulating EPCs were identified via flow cytometry analysis (FCA) and immunofluorescence microscopy. CD $45^{-}$(FITC) $/ \mathrm{CD} 34^{+}(\mathrm{PE}-\mathrm{Cy} 7) / \mathrm{KDR}^{+}$(APC) (cat. nos. FHF045-025, FHN034-025 and FHK309-025, respectively; all Beijing 4A Biotech Co., Ltd.) were defined as EPCs by FCA. Dil-acLDL ${ }^{+} /$FITC-lectin ${ }^{+}$were defined as EPCs by immunofluorescence microscopy. FCA and immunofluorescence microscopy were performed as previously described (19-26).

Migration and proliferation ability of EPCs. EPC migration was evaluated as previously described (19-22). Proliferation activity was assessed via MTT assay (cat. no. ab211091; Abcam) according to the manufacturer's instructions and our previous studies $(19,20,22,27,28)$.

Evaluation of EPC adhesive activity. EPCs were digested with Parenzyme (cat. no. CCS001; Tetra-n-Bo Biotechnology Co., Ltd.) at $37^{\circ} \mathrm{C}$ for $1 \mathrm{~min}$ and resuspended in culture medium to a final density of $2 \times 10^{5} / \mathrm{ml}$. A total of $500 \mu 1\left(2 \times 10^{5} / \mathrm{ml}\right.$ per well $)$ cell suspension was inoculated in 24-well plates precoated with fibronectin (cat. no. 5050-1MG; Tetra-n-Bo Biotechnology Co., Ltd.) and incubated at $37^{\circ} \mathrm{C}$ for $30 \mathrm{~min}$. After being washed twice with PBS, EPCs were fixed with $4 \%$ polyformic acid for $10 \mathrm{~min}$ at $37^{\circ} \mathrm{C}$. After two further washes with PBS, adherent EPCs were observed under an inverted immunofluorescence microscope (magnification, x100). Adherent cells were counted in three randomly selected fields of view.

Measurement of plasma NO levels and NO secretion by EPCs. NO levels were measured using the NO levels test kit (cat. no. A012-1-2; Nanjing Jiancheng Bioengineering Institute) according to the manufacturer's instructions.

FMD and nitroglycerin-mediated dilation (NMD). FMD was measured as previously described $(29,30)$. For NMD, pressure in an upper-forearm sphygmomanometer cuff was raised to $250 \mathrm{mmHg}$ for $5 \mathrm{~min}$. FMD was calculated as the percentage increase in mean diastolic diameter after reactive hyperaemia at 55-65 sec after deflation to baseline. NMD was calculated as follows: at 15 min after FMD measurement, $400 \mu \mathrm{g}$ sublingual nitroglycerin was administered and after a further $5 \mathrm{~min}$, the diastolic diameter was remeasured to assess endothelial-independent dilatation.

Western blot analysis. Western blot analysis was performed as previously described (18). Briefly, protein concentrations 
Table I. Clinical and biochemical characteristics.

\begin{tabular}{|c|c|c|c|c|}
\hline Characteristic & Control $(n=20)$ & Low-risk $(n=20)$ & Middle-risk $(\mathrm{n}=20)$ & High-risk $(n=20)$ \\
\hline Age, years & $55.30 \pm 12.90$ & $55.90 \pm 13.70$ & $56.70 \pm 13.50$ & $57.40 \pm 14.50$ \\
\hline Weight, kg & $70.40 \pm 5.90$ & $70.80 \pm 6.40$ & $71.40 \pm 6.20$ & $70.90 \pm 6.50$ \\
\hline AST, mmol/l & $70.80 \pm 8.40$ & $71.00 \pm 7.90$ & $71.80 \pm 7.50$ & $72.40 \pm 7.10$ \\
\hline ALT, mmol/1 & $25.60 \pm 6.40$ & $26.90 \pm 6.10$ & $26.70 \pm 6.10$ & $25.40 \pm 6.90$ \\
\hline BUN, mmol/l & $23.50 \pm 8.90$ & $24.90 \pm 6.80$ & $23.40 \pm 7.10$ & $24.00 \pm 7.30$ \\
\hline $\mathrm{Cr}, \mathrm{mmol} / \mathrm{l}$ & $5.60 \pm 1.30$ & $5.40 \pm 1.70$ & $5.60 \pm 1.80$ & $5.70 \pm 1.50$ \\
\hline $\mathrm{LDL}, \mathrm{mmol} / \mathrm{l}$ & $70.30 \pm 13.50$ & $71.30 \pm 13.90$ & $72.80 \pm 11.60$ & $72.90 \pm 16.90$ \\
\hline $\mathrm{TC}, \mathrm{mmol} / \mathrm{l}$ & $2.80 \pm 0.56$ & $2.90 \pm 0.58$ & $2.95 \pm 0.59$ & $3.15 \pm 0.43$ \\
\hline HDL, mmol/1 & $4.83 \pm 0.52$ & $5.02 \pm 0.51$ & $4.97 \pm 0.54$ & $5.07 \pm 0.50$ \\
\hline $\mathrm{TG}, \mathrm{mmol} / \mathrm{l}$ & $1.36 \pm 0.28$ & $1.42 \pm 0.27$ & $1.43 \pm 0.24$ & $1.44 \pm 0.21$ \\
\hline CRP, mmol/l & $102.10 \pm 20.70$ & $103.00 \pm 23.90$ & $107.20 \pm 22.60$ & $104.70 \pm 23.40$ \\
\hline $\mathrm{HR}$, beats/min & $72.30 \pm 8.50$ & $71.80 \pm 9.20$ & $73.30 \pm 7.80$ & $73.10 \pm 9.30$ \\
\hline BMI & $23.10 \pm 2.30$ & $23.50 \pm 2.20$ & $23.30 \pm 2.50$ & $23.40 \pm 2.70$ \\
\hline $\mathrm{SBP}, \mathrm{mmHg}$ & $119.20 \pm 10.50$ & $118.90 \pm 11.10$ & $119.80 \pm 10.70$ & $120.30 \pm 9.90$ \\
\hline $\mathrm{DBP}, \mathrm{mmHg}$ & $69.50 \pm 5.00$ & $70.90 \pm 4.80$ & $71.90 \pm 3.80$ & $70.50 \pm 5.50$ \\
\hline
\end{tabular}

Data are presented as the mean $\pm \mathrm{SD}$. ALT, alanine aminotransferase; AST, aspartate aminotransferase; BUN, serum urea nitrogen; Cr, creatinine; CRP, C-reactive protein; LDL, low-density lipoprotein; TC, total cholesterol; HDL, high-density lipoprotein; HR, heart rate; BMI, body mass index; SBP, systolic blood pressure; DBP, diastolic blood pressure; TG, triglyceride.

were determined using the BCA method. Proteins $(50 \mu \mathrm{g})$ were separated via 5\% SDS-PAGE. Following blocking with $5 \%$ skimmed milk powder was used at $4^{\circ} \mathrm{C}$ for $12 \mathrm{~h}$, the membranes were incubated at $4^{\circ} \mathrm{C}$ for $12 \mathrm{~h}$ with the following primary antibodies: $\beta$-actin (1:2,000; cat. no. 3700T; Cell Signaling Technology Inc.), endothelial NO synthase (eNOS; 1:1,000; cat. no. 9575; Cell Signaling Technology, Inc.) and phosphorylated (p)-eNOS (1:1,000; cat. no. 9571; Cell Signaling Technology, Inc.). Subsequently, the membranes were incubated with a HRP-conjugated secondary antibody (1:3,000; cat. no. 7074; Cell Signaling Technology, Inc.) at room temperature for $2 \mathrm{~h}$. Protein expression was semi-quantified using Image J software (version 1.0; National Institutes of Health) with $\beta$-actin as the loading control.

Statistical analysis. Data are presented as the mean \pm SD from three independent experiments. Data were analyzed using GraphPad Prism 6.0 software (GraphPad Software, Inc.). Comparisons between multiple groups were analyzed by one-way ANOVA and post hoc Tukey's test. The persons who analyzed the data were blinded to group assignment. Univariate correlations were calculated using Pearson's coefficient (r). $\mathrm{P}<0.05$ was considered to indicate a statistically significant difference.

\section{Results}

Patient characteristics. Patient characteristics are presented in Table I. Age, BMI, levels of cholesterol, high- and low-density lipoprotein, triglycerides, plasma glucose, and high-sensitivity $\mathrm{C}$-reactive protein were not significantly different between groups.
Levels and activity of circulating EPCs. EPCs were evaluated by fluorescence activated cell sorting analysis (Fig. S1A) and phase-contrast fluorescent microscope labeled by Dil-LDL and FITC-lectin (Fig. S1B).

Number of circulating EPCs is shown in Fig. 1A and B. The levels of circulating EPCs evaluated by FCA (Fig. S2) and immunofluorescence microscopy in patients with CAD were significantly lower compared with the control group. The number of circulating EPCs in middle- and high-risk groups were decreased compared with the low-risk group. The difference between middle- and high-risk groups was also significant.

Migratory activity of EPCs in patients with CAD was decreased compared with the control group $(\mathrm{P}<0.01$; Figs. 1C and S3). Similarly, the proliferative and adhesive activity (Fig. S4) of circulating EPCs in patients with CAD were significantly impaired compared with control group (both $\mathrm{P}<0.01$; Fig. $1 \mathrm{D}$ and $\mathrm{E}$ ). The migratory activity of circulating EPCs in middle- and high-risk group were lower than in the low-risk group. The migratory activity of circulating EPCs was also significantly different between middle- and high-risk groups. Similar results were observed for the proliferative and adhesive function of circulating EPCs (Fig. 1D and E).

FMD and NMD. FMD in patients with CAD decreased compared with the control group (Fig. 2A). The FMD in middle- and high-risk group was lower than in the low-risk group. FMD was also significantly different between the middle- and high-risk groups. NMD (Fig. 2B) showed no significant difference between groups.

Plasma NO levels and secretion by EPCs. Plasma NO levels in patients with CAD were significantly lower than in the Control group (Fig. 2C). The plasma NO levels in the middle- and 

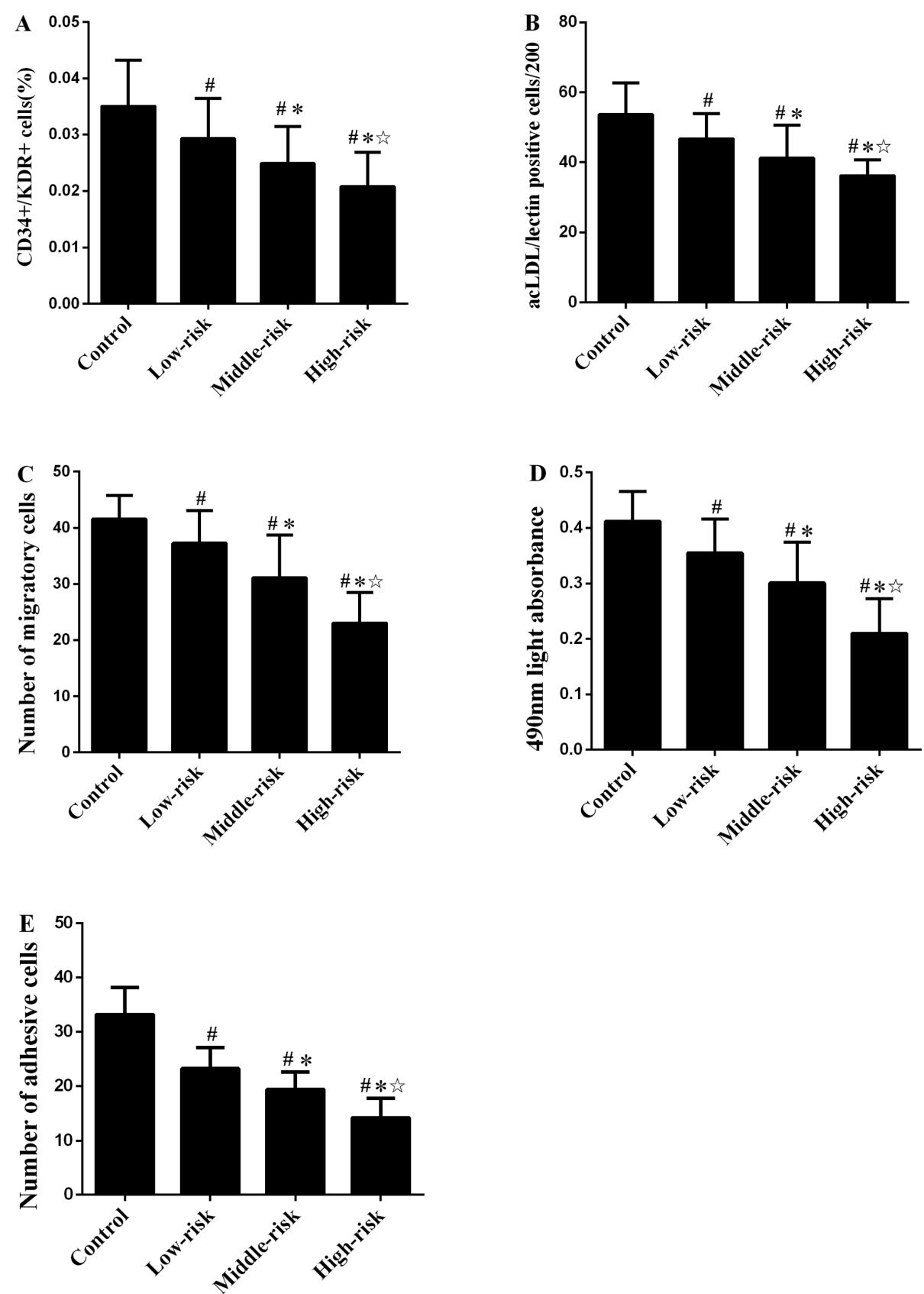

Figure 1. Number of circulating EPCs. EPCs were evaluated by (A) fluorescence activated cell sorting analysis and (B) phase-contrast fluorescent microscopy. Levels of circulating EPCs in patients with coronary artery disease were statistically different from those in the Control group. The number of EPCs in middleand high-risk group were decreased compared with the low-risk group. The differences were significant between middle- and high-risk group. Similar results were observed for (C) migration, (D) proliferation and (E) adhesion. Data are presented as the mean $\pm \mathrm{SD} .{ }^{*} \mathrm{P}<0.05$ vs. Control. ${ }^{\text {P }}<0.05$ vs. low-risk. ${ }^{\text {" }} \mathrm{P}<0.05$ vs. middle-risk. EPC, endothelial progenitor cells.

high-risk groups were lower than in the low-risk group. The plasma NO levels were also significantly different between the middle- and high-risk groups. Similar results were detected for NO secretion by EPCs (Fig. 2D).

Number and activity of circulating EPCs in groups with different numbers of coronary artery lesions. The number of circulating EPCs evaluated by FCA (Figs. 3A and S5) and immunofluorescence microscopy (Figs. 3B and S1B) in patients with $\mathrm{CAD}$ was significantly decreased compared with the Control group. The number of circulating EPCs in multiple (double or triple) vessel groups was decreased compared with the single vessel group. There was also a significant difference in the number of circulating EPCs between the double and triple vessel groups. Similar numbers of migratory, proliferative and adhesive (Fig. 3C-E) circulating EPCs were observed in groups with different number coronary artery lesions.

FMD and NMD in groups with different numbers of coronary artery lesions. FMD in patients with CAD decreased compared with that in the Control group (Fig. 4A). FMD in multiple (double or triple) vessels group was decreased compared with single vessel group. There was also a significant difference in FMD between the double and triple vessels. NMD showed no 

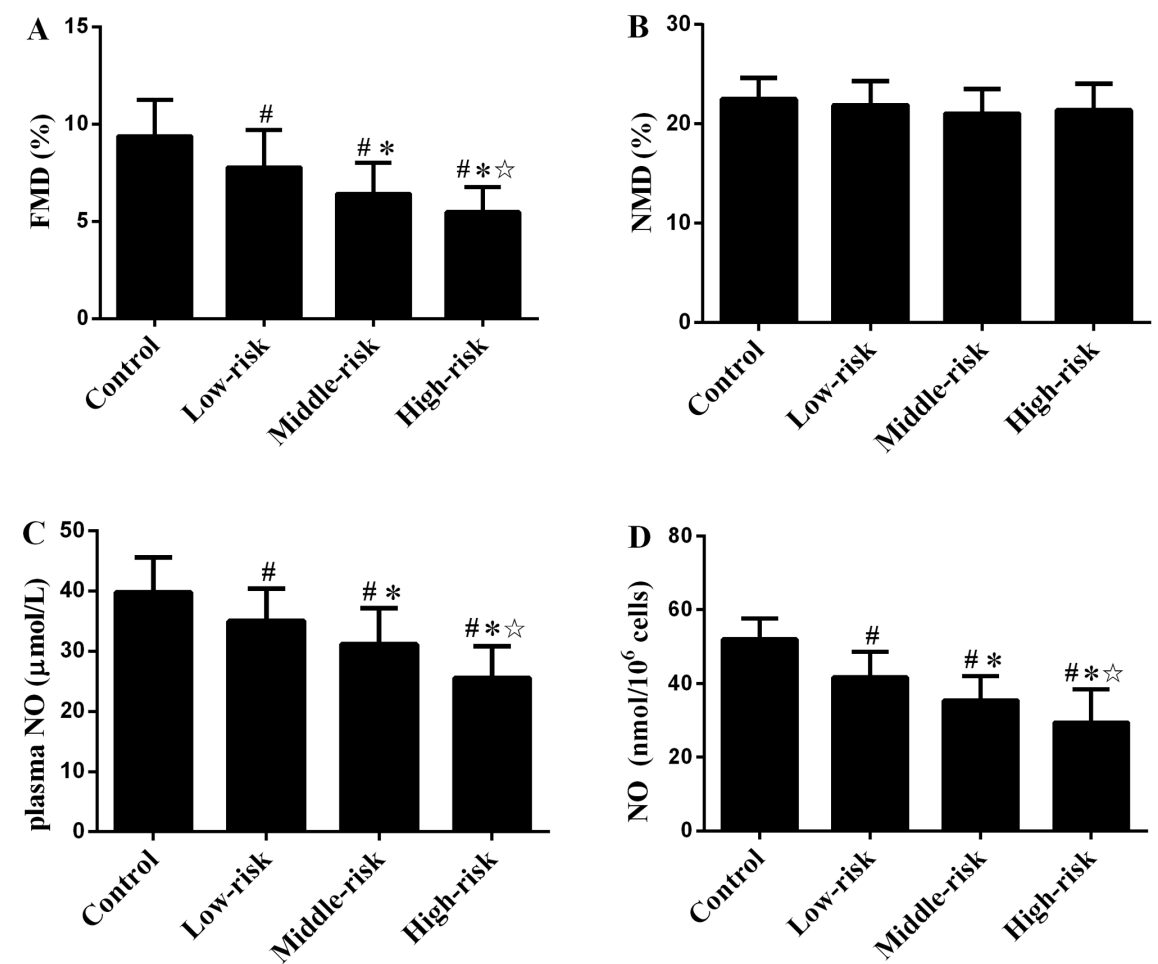

Figure 2. Endothelial function in different group. (A) FMD in patients with coronary artery disease was statistically different from that in Control group. FMD in the middle- and high-risk groups were decreased compared with the low-risk group. Differences in FMD were significant between middle- and high-risk groups. (B) NMD was not significantly different between groups. NO (C) plasma levels and (D) secretion by endothelial progenitor cells. Data are presented as the mean \pm SD. ${ }^{~} \mathrm{P}<0.05$ vs. Control. "P $<0.05$ vs. low-risk. "P $<0.05$ vs. middle-risk. FMD, flow-mediated dilatation; NMD, nitroglycerin-mediated dilation; NO, nitric oxide.

significant difference between groups with different numbers of coronary artery lesions (Fig. 4B).

NO plasma levels and secretion by EPCs in groups with different numbers of coronary artery lesions. Plasma NO levels in patients with CAD were significantly lower than in the Control group (Fig. 4C). The plasma NO levels in multiple (double or triple) vessel groups were decreased compared with the single vessel group. There was also a significant difference in plasma NO levels between the double and triple vessel groups. Similar results were observed in NO secretion by EPCs in groups with different number of coronary artery lesions (Fig. 4D).

Western blot results. eNOS and p-eNOS protein expression levels of circulating EPCs were determined in groups with different Syntax scores and numbers of coronary lesion (Fig. 5A and B). eNOS expression levels were not significantly different, whereas p-eNOS protein expression levels were decreased in the CADs group compared with the Control group. p-eNOS protein expression levels in the middle- and high-risk groups were decreased compared with the low-risk group; there were also significant differences between the high- and middle-risk groups. eNOS expression were not significantly different between groups with different numbers of coronary artery lesions, whereas p-eNOS protein expression levels were decreased in the CAD groups compared with the Control group (Fig. 5B). p-eNOS protein expression levels in the multiple vessel groups were decreased compared with the single vessel group and there was a significant difference between the triple and double vessel groups.
Correlation between circulating EPC levels and activity and Syntax score. A negative univariate correlation between the number of circulating EPCs and Syntax score (Fig. 6A and B) was detected. Both were evaluated by FCA (Fig. 6A; $\mathrm{R}=0.51 ; \mathrm{P}<0.01$ ) and cell culture (Fig. $6 \mathrm{~B} ; \mathrm{R}=0.55 ; \mathrm{P}<0.01$ ). The numbers of migratory (Fig. 6C; $\mathrm{R}=0.48$ ), proliferative (Fig. 6D; R=0.54) and adhesive EPCs (Fig. 6E; R=0.58) was significantly inversely correlated with the Syntax score (all $\mathrm{P}<0.01)$.

Correlation between FMD, NMD and Syntax score. There was a significant inverse correlation between FMD and Syntax score (Fig. 7A; $\mathrm{R}=0.66 ; \mathrm{P}<0.01$ ). There was no significant correlation between NMD and Syntax score (Fig. 7B; R=0.01; $\mathrm{P}>0.05)$.

Correlation between NO plasma levels or secretion by EPCs and Syntax score. Both NO plasma levels and secretion by EPCs were negatively correlated with Syntax score (Fig. 7C and D; $\mathrm{R}=0.58$ and $\mathrm{R}=0.63$, respectively; both $\mathrm{P}<0.01$ ).

Correlation between circulating EPCs and the number of coronary lesions. A strong negative univariate correlation was detected between circulating EPCs number and number of coronary lesions, as evaluated by FCA (Fig. 8A; R=0.48; $\mathrm{P}<0.01$ ) and immunofluorescence microscopy (Fig. 8B; $\mathrm{R}=0.49 ; \mathrm{P}<0.01$ ). The migratory (Fig. $8 \mathrm{C} ; \mathrm{R}=0.75$ ), proliferative (Fig. 8D; R=0.72) and adhesive activity (Fig. 8E; R=0.63) exhibited a significant inverse correlation with the number of coronary lesions (all $\mathrm{P}<0.01$ ). 

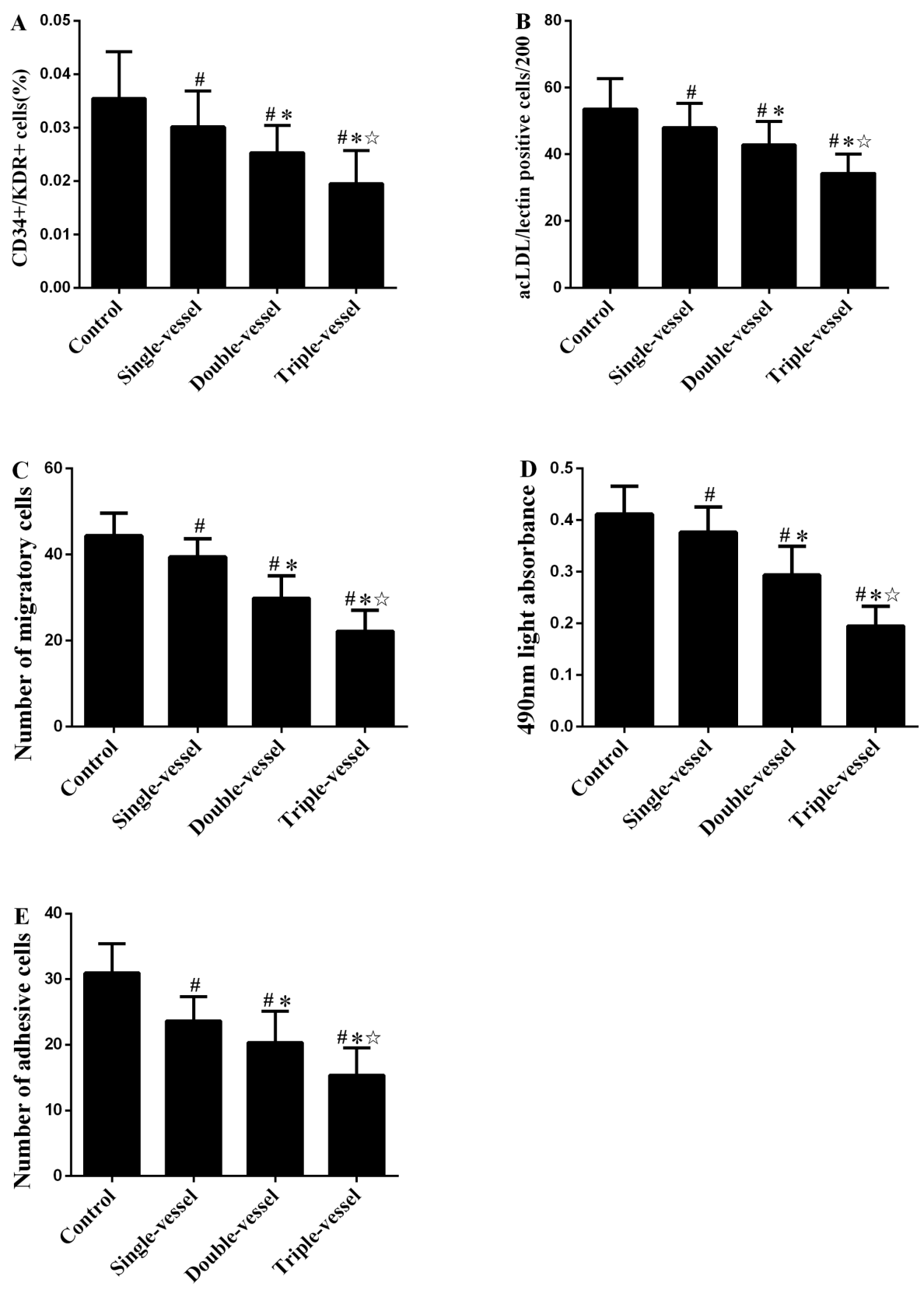

Figure 3. Number and activity of EPCs in groups with different numbers of coronary lesions. Number of circulating EPCs in groups with different numbers of coronary lesion evaluated by (A) fluorescence activated cell sorting analysis and (B) phase-contrast fluorescent microscopy. Levels of circulating EPCs in patients with coronary artery disease were statistically lower compared with the Control group. The number of circulating EPCs in multiple (double or triple) vessel groups were decreased compared with the single-vessel group. The differences were significant between double and triple vessel groups. Similar results were observed for EPC (C) migration, (D) proliferation and (E) adhesion. Data are presented as the mean $\pm \mathrm{SD}$. "P<0.05 vs. Control. "P<0.05 vs. single vessel. "P $<0.05$ vs. double vessel. EPCs, endothelial progenitor cells.

Correlation between FMD, NMD and the number of coronary lesions. There was a significant inverse correlation between FMD and the number of coronary lesions (Fig. 9A; R=0.54; $\mathrm{P}<0.01)$. By contrast, there was no significant correlation between NMD and the number of coronary lesions (Fig. 9B; $\mathrm{R}=0.04 ; \mathrm{P}>0.05$ ).

Correlation between NO plasma levels or secretion by EPCs and the number of coronary lesions. Both NO plasma levels and secretion by EPCs were negatively correlated with the number of coronary lesions (Fig. 9C and D; R=0.55 and $\mathrm{R}=0.71$, respectively; both $\mathrm{P}<0.01$ ).

\section{Discussion}

The present study showed that the number and function of circulating EPCs, as well as FMD, were impaired in patients with CAD with a higher Syntax score. Similar alterations were observed in NO levels in plasma or secreted by EPCs into culture medium. In addition, the number and activity of EPCs, FMD, and NO levels in plasma or secreted by EPCs were attenuated in multiple vessel groups compared with the single vessel group. There were significant inverse correlations between the number and activity of circulating EPCs and endothelial function and Syntax score. The NO plasma levels 

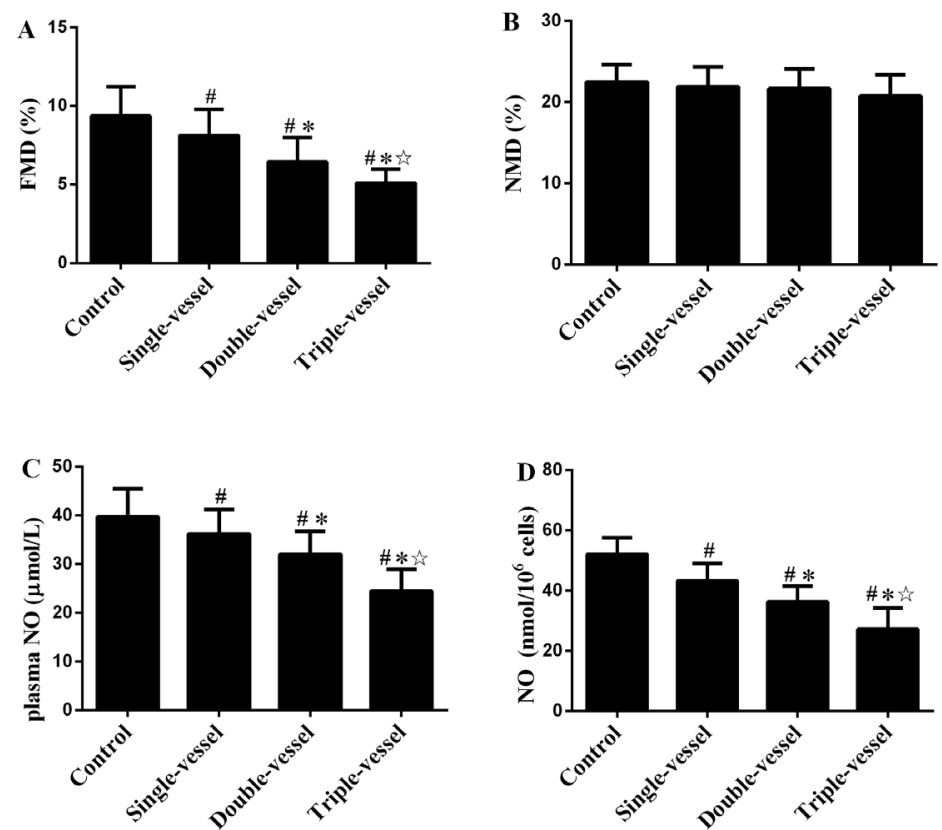

Figure 4. Endothelial function of EPCs in groups with different numbers of coronary lesions. (A) FMD in patients with coronary artery disease was decreased compared with that in the Control group. FMD in multiple (double or triple) vessel groups was decreased compared with the single vessel group. The differences in FMD were significant between double and triple vessel groups. (B) NMD was not significantly different between groups. NO (C) plasma levels and (D) secretion by endothelial progenitor cells. Data are presented as the mean $\pm \mathrm{SD}$. ${ }^{*} \mathrm{P}<0.05$ vs. Control. ${ }^{*} \mathrm{P}<0.05$ vs. single vessel. " $\mathrm{P}<0.05$ vs. double vessel. FMD, flow-mediated dilatation; NMD, nitroglycerin-mediated dilation; NO, nitric oxide.

A
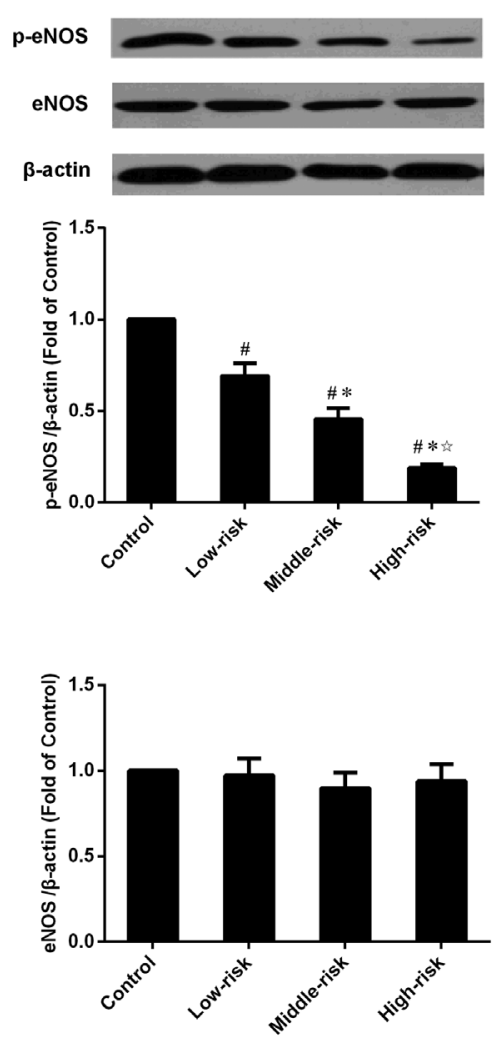

B
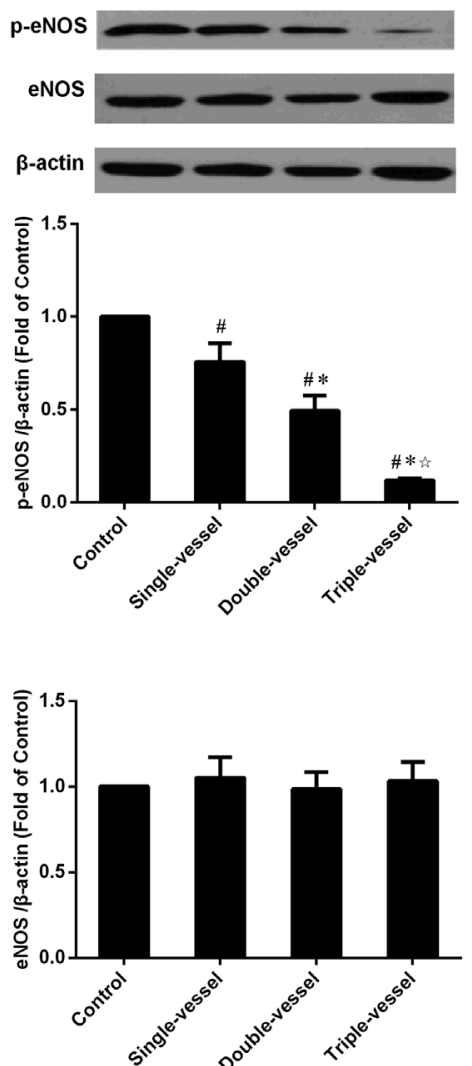

Figure 5. eNOS protein expression levels of circulating EPCs from patients with CAD with different Syntax scores. (A) eNOS protein expression levels were not statistically different between different risk groups. p-eNOS protein expression of circulating EPCs in CAD groups was decreased compared with the Control group. p-eNOS protein expression levels in the middle- and high-risk groups were decreased compared with the low-risk group. The differences were significant between middle-group and high-risk group. eNOS protein expression levels were not statistically different between groups with different numbers of coronary lesions. (B) p-eNOS protein expression in multiple (double or triple) vessel groups were decreased compared with the single vessel group. A significant difference was observed between the double and triple vessel groups. ${ }^{~} \mathrm{P}<0.05$ vs. Control. ${ }^{*} \mathrm{P}<0.05$ vs. low-risk or single vessel. ${ }^{*} \mathrm{P}<0.05$ vs. middle-risk or double vessel. eNOS, endothelial nitric oxide synthase; EPCs, endothelial progenitor cells; CAD, coronary artery disease; p-, phosphorylated. 

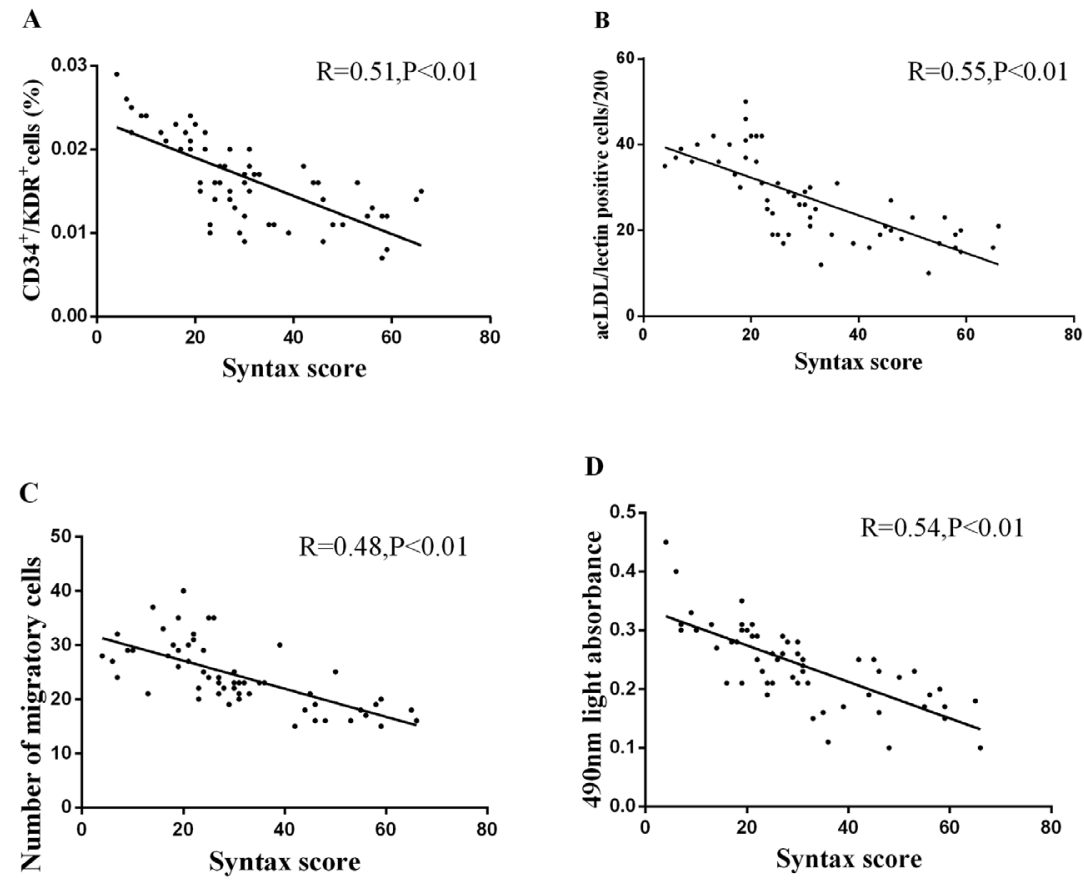

$\mathbf{E}$

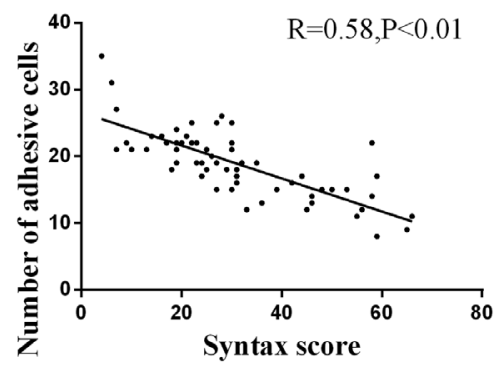

Figure 6. Correlation between number and activity of circulating EPCs, endothelial function and Syntax score. (A and B) There was a negative correlation between the number of circulating EPCs and Syntax score. Correlation between numbers of (C) migratory, (D) proliferative and (E) adhesive EPCs and Syntax score. EPCs, endothelial progenitor cells.
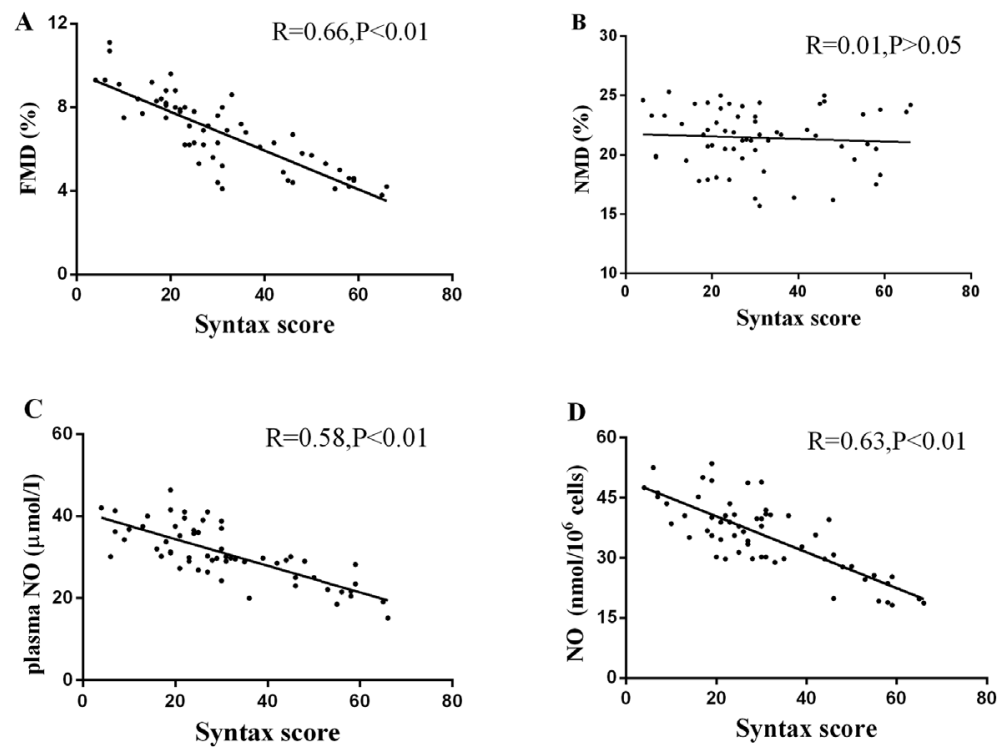

Figure 7. Correlations between Syntax score and FMD, NMD, plasma NO or NO secretion by endothelial progenitor cells. (A) Negative correlation between FMD and Syntax score. (B) No correlation between NMD and Syntax score was observed. Correlation between NO (C) plasma levels and (D) secretion by endothelial progenitor cells and Syntax score. FMD, flow-mediated dilatation; NMD, nitroglycerin-mediated dilation; NO, nitric oxide. 

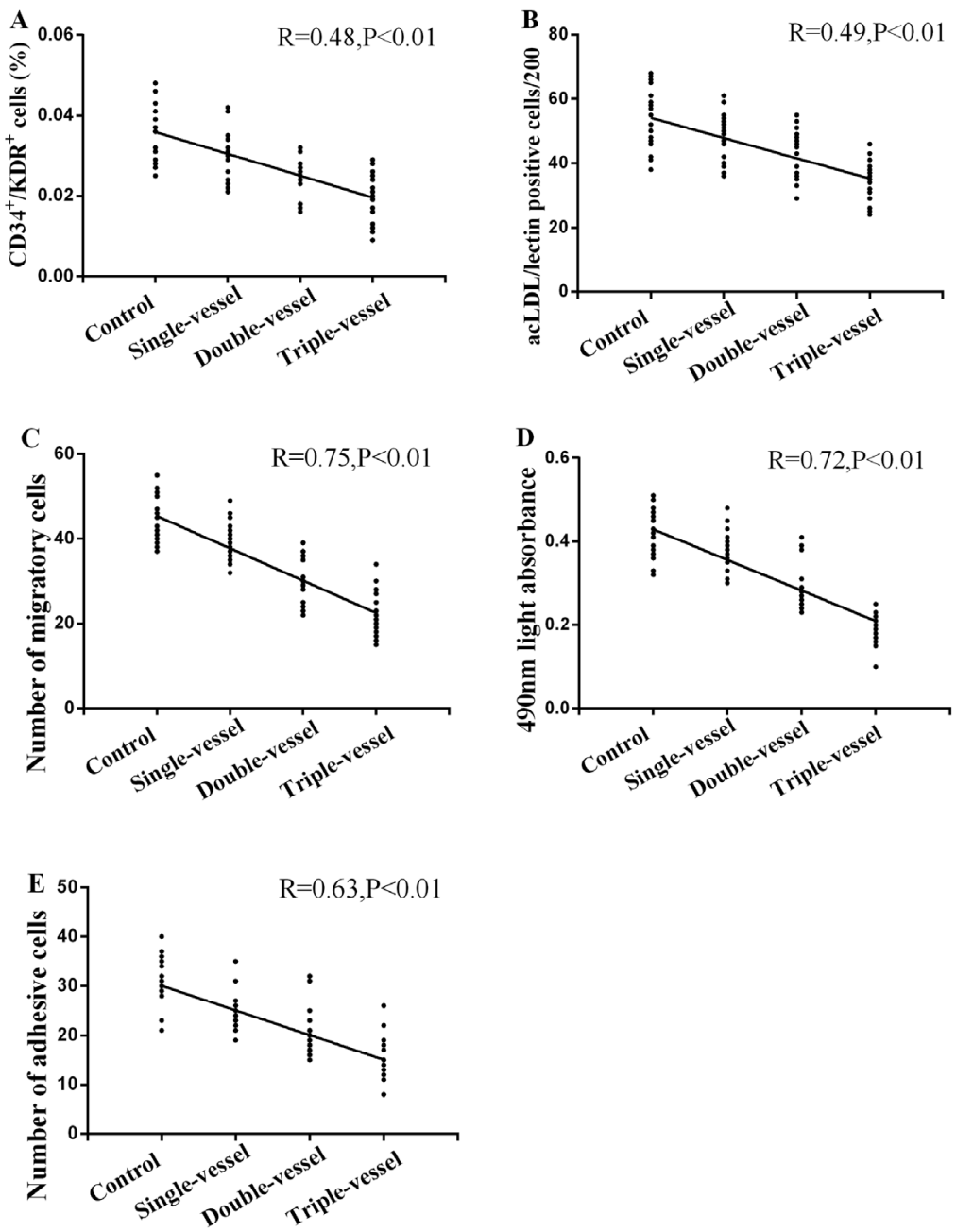

Figure 8. Correlation between the number or activity of circulating EPCs or endothelial function and the number of coronary lesions. (A and B) Negative correlation between levels of circulating EPCs and the number of coronary lesions. Similar results were observed for (C) migratory, (D) proliferative and (E) adhesive EPCs. EPCs, endothelial progenitor cells.

and secretion by EPCs were also negatively correlated with Syntax score.

Syntax score is a risk stratification tool for patients with CAD based on the anatomy of the coronary artery, providing a quantifiable objective evaluation of complexity and severity for coronary artery lesions (31-34). The primary cause of CAD is atherosclerosis, which is characterized by defective endothelial function and plaque formation in the inner wall of the artery (35). The present study revealed that FMD decreased in patients with high Syntax score, indicating that endothelial function was impaired and was associated with greater severity and complexity of coronary artery lesions.

A previous study showed that the number and activity of circulating EPCs are decreased in patients with CAD (5), suggesting that attenuated endogenous endothelial repair capacity is involved in coronary artery abnormalities. Studies have reported that the number of circulating EPCs is inversely correlated with Syntax score $(6,7)$, suggesting that the quantitative alteration in circulating EPCs may be a cytobiological parameter to evaluate the severity and complexity of CAD. However, the association between activity of EPCs, as well as endothelial function, and Syntax score is not clear.
Prior studies revealed a correlation between endothelial function and the number and activity of circulating EPCs $(17,18)$. In light of the association between impaired endothelial function and high Syntax score, it was hypothesized that the activity of circulating EPCs is decreased in patients with high Syntax score. Here, the migratory, proliferative and adhesive function of circulating EPCs were shown to be attenuated in patients with a higher Syntax score, indicating that the quantitative and qualitative alteration in circulating EPCs is a surrogate parameter to evaluate the severity and complexity of CAD. These data provide evidence for the potential role of endothelial function and capacity for endogenous repair of endothelial injury in assessment of anatomical abnormalities of the coronary artery. The migration, proliferation and adhesion capability of EPCs, which are characteristic of morphological vascular changes $(4,5)$, were measured and analyzed. FMD is an indicator of vascular functional change (36). Here, the characteristics of vascular morphological and functional changes were assessed to investigate the correlation between functional change and risk levels.

Endothelial function and circulating EPCs were also correlated with the number of lesioned vessels. Compared with single vessel lesions, multiple vessel lesions resulted in 

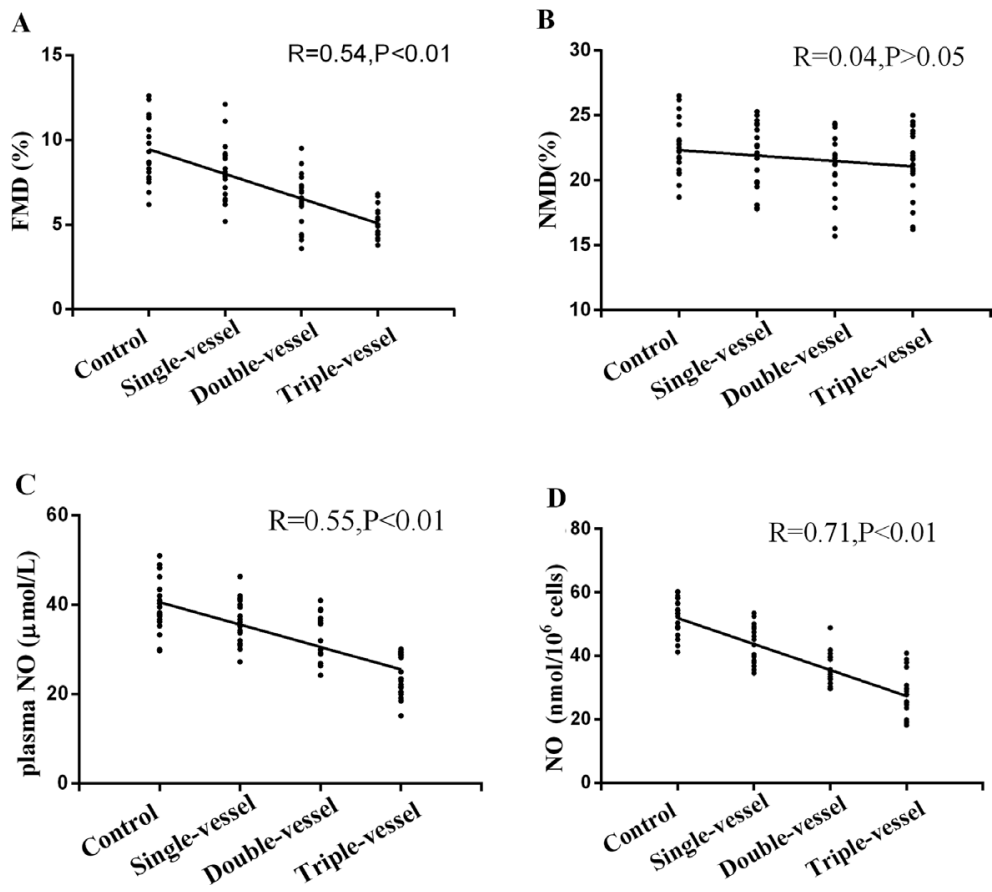

Figure 9. Correlation between FMD, NMD and number of coronary lesions. (A) There was a negative correlation between the FMD and the number of coronary lesions. (B) NMD was not correlated with the number of coronary lesions. Correlation between NO (C) plasma levels and (D) secretion by endothelial progenitor cells and number of coronary lesions. FMD, flow-mediated dilatation; NMD, nitroglycerin-mediated dilation; NO, nitric oxide.

decreased numbers or activity of circulating EPCs, FMD and NO levels in plasma or secreted by EPCs. This is similar to the correlations with Syntax score, which is primarily based on the anatomy of the coronary artery and includes the number of vessel lesions and severity of lesions.

NO serves an important role in regulating endothelial function and the number and function of circulating EPCs. eNOS is the NOS isoform responsible for the production of NO and key regulators of mobilization and function of EPCs $(17,18)$. In the present study, levels of eNOS in circulating EPCs were preserved in patients with CAD but levels of p-eNOS were decreased compared with the Control group. These results indicate that the decreased phosphorylation of eNOS, but not the alteration of eNOS expression levels, contributed to decreased NO secretion by circulating EPCs in patients with CAD. In addition, phosphorylation of eNOS was lower in patients with high a Syntax score compared with the low Syntax score group. Furthermore, patients with CAD with multiple vessel lesions also exhibited notably decreased eNOS phosphorylation. Therefore, it was inferred that decreased phosphorylation of eNOS may be the mechanism underlying decreased NO in circulating EPCs and FMD in patients with CAD.

In addition, the present study showed a negative correlation between Syntax score and the number and activity of circulating EPCs, as well as endothelial function, further indicating that impaired repair capacity of endothelial injury and subsequent decreased endothelial function is accompanied by serious and complicated coronary artery anatomical abnormalities. NO serves an important role in regulating endothelial function and the number and function of circulating EPCs (37-39). There was an inverse correlation between NO production and Syntax score, suggesting that decreased NO bioavailability may be the mechanism underlying decreased levels of circulating EPCs and endothelial dysfunction in patients with CAD with a high Syntax score.

The present study may have important implications. First, the present data revealed that decreased FMD was associated with high Syntax score, indicating endothelial function may be a clinical indicator of coronary artery anatomical abnormalities. Second, these results showed that decreased number or activity of circulating EPCs was associated with a higher Syntax score, demonstrating that circulating EPCs may serve as an important surrogate biomarker for evaluation of the severity of CAD. Finally, decreased NO biosynthesis may be an important mechanism underlying impaired endothelial repair capacity and dysfunction in patients with a high Syntax score. Therefore, strategies to increase NO production, such as exercise and statin therapy, may be a novel therapeutic approach for endothelial injury in patients with CAD.

To the best of our knowledge, the present study is the first to demonstrate that the number and activity of circulating EPCs, as well as FMD, are negatively correlated with Syntax score, indicating that circulating EPCs and endothelial function may be key surrogate biomarkers for the evaluation of severity and complexity of CAD. Enhancing the number or activity of circulating EPCs and improving endothelial function may be significant therapeutic approaches for serious and complicated CAD.

\section{Acknowledgements}

The abstract was presented at the 29th Great Wall International Cardiology Conference Oct 11-14 in Beijing, China and published as abstract in Journal of the American College of 
Cardiology, 2018 Volume 72, Issue 16, Supplement, 16 October 2018, Page C8.

\section{Funding}

The present study was financially supported by the projects of the Fundamental Research Funds of Jiangmen Central Hospital (grant no. D201901), the Outstanding Youth Fund Projects of Jiangmen Central Hospital (grant no. J202003) and Jiangmen City Science and Technology Plan (grant nos. 2020YLA100 and 2020YLA133).

\section{Availability of data and materials}

The datasets used and/or analyzed during the current study are available from the corresponding author on reasonable request.

\section{Authors' contributions}

BZ and DL performed the experiments, analyzed data and wrote the manuscript. GL and WT performed statistical analysis and discussion and investigated study subjects. GZ and JL designed the study and reviewed the manuscript. BZ and GZ confirm the authenticity of all the raw data. All authors read and approved the final manuscript.

\section{Ethics approval and consent to participate}

The present study was approved by the Jiangmen Central Hospital Ethics Committee on Research on Humans (approval no. 20170419). Written informed consent was obtained from all participants.

\section{Patient consent for publication}

Not applicable.

\section{Competing interests}

The authors declare that they have no competing interests.

\section{References}

1. He Y, Kothari V and Bornfeldt KE: High-density lipoprotein function in cardiovascular disease and diabetes mellitus. Arterioscler Thromb Vasc Biol 38: e10-e16, 2018.

2. Piccolo R, Giustino G, Mehran R and Windecker S: Stable coronary artery disease: Revascularisation and invasive strategies. Lancet 386: 702-713, 2015.

3. Calderón-Gerstein WS, López-Peña A, Macha-Ramírez R, Bruno-Huamán A, Espejo-Ramos R, Vílchez-Bravo S, Ramírez-Breña M, Damián-Mucha $\mathrm{M}$ and Matos-Mucha A: Endothelial dysfunction assessment by flow-mediated dilation in a high-altitude population. Vasc Health Risk Manag 13: 421-426, 2017.

4. Aicher A, Zeiher AM and Dimmeler S: Mobilizing endothelial progenitor cells. Hypertension 45: 321-325, 2005.

5. Hill JM, Zalos G, Halcox JP, Schenke WH, Waclawiw MA, Quyyumi AA and Finkel T: Circulating endothelial progenitor cells, vascular function, and cardiovascular risk. N Engl J Med 348: 593-600, 2003

6. Ghem C, Dias LD, Sant'Anna RT, Kalil RAK, Markoski M and Nardi NB: Combined analysis of endothelial, hematopoietic, and mesenchymal stem cell compartments shows simultaneous but independent effects of age and heart disease. Stem Cells Int 2017: 5237634,2017
7. Chi J, Hong X, Wang Y, Zhao J and Yang W: Inverse correlation between circulating endothelial progenitor cells with CD $34^{+} \mathrm{CD} 133^{+}$and the severity of coronary atherosclerosis assessed by Syntax score. Am J Med Sci 347: 457-462, 2014.

8. Kolh P and Windecker S: ESC/EACTS myocardial revascularization guidelines 2014. Eur Heart J 35: 3235-3236, 2014.

9. Sianos G, Morel MA, Kappetein AP, Morice MC, Colombo A Dawkins K, van den Brand M, Van Dyck N, Russell ME, Mohr FW and Serruys PW: The Syntax score: An angiographic tool grading the complexity of coronary artery disease. EuroIntervention 1: 219-227, 2005 .

10. Tanaka T, Seto S, Yamamoto K, Kondo M and Otomo T: An assessment of risk factors for the complexity of coronary artery disease using the Syntax score. Cardiovasc Interv Ther 28: 16-21, 2013.

11. Wykrzykowska JJ, Garg S, Girasis C, de Vries T, Morel MA, van Es GA, Buszman P, Linke A, Ischinger T, Klauss V, et al: Value of the Syntax score for risk assessment in the all-comers population of the randomized multicenter LEADERS (limus eluted from A durable versus ERodable stent coating) trial. J Am Coll Cardiol 56: 272-277, 2010.

12. Mohr FW, Morice MC, Kappetein AP, Feldman TE, Ståhle E, Colombo A, Mack MJ, Holmes DR Jr, Morel MA, Van Dyck N, et al: Coronary artery bypass graft surgery versus percutaneous coronary intervention in patients with three-vessel disease and left main coronary disease: 5-year follow-up of the randomised, clinical Syntax trial. Lancet 381: 629-638, 2013.

13. Mohr FW, Rastan AJ, Serruys PW, Kappetein AP, Holmes DR, Pomar JL, Westaby S, Leadley K, Dawkins KD and Mack MJ: Complex coronary anatomy in coronary artery bypass graft surgery: Impact of complex coronary anatomy in modern bypass surgery? Lessons learned from the Syntax trial after two years. J Thorac Cardiovasc Surg 141: 130-140, 2011.

14. Aicher A, Heeschen C, Mildner-Rihm C, Urbich C, Ihling C, Technau-Ihling K, Zeiher AM and Dimmeler S: Essential role of endothelial nitric oxide synthase for mobilization of stem and progenitor cells. Nat Med 9: 1370-1376, 2003.

15. Duda DG, Fukumura D and Jain RK: Role of eNOS in neovascularization: NO for endothelial progenitor cells. Trends Mol Med 10: 143-145, 2004.

16. Urbich C and Dimmeler S: Endothelial progenitor cells: characterization and role in vascular biology. Circ Res 95: 343-353, 2004.

17. Zhen Y, Xiao S, Ren Z, Shen HW, Su H, Tang YB and Zeng H: Increased endothelial progenitor cells and nitric oxide in young prehypertensive women. J Clin Hypertens (Greenwich) 17: 298-305, 2015.

18. Zeng H, Jiang Y, Tang H, Ren Z, Zeng G and Yang Z: Abnormal phosphorylation of Tie2/Akt/eNOS signaling pathway and decreased number or function of circulating endothelial progenitor cells in prehypertensive premenopausal women with diabetes mellitus. BMC Endocr Disord 16: 13, 2016.

19. Yang Z, Chen L, Su C, Xia WH, Wang Y, Wang JM, Chen F, Zhang YY, Wu F, Xu SY, et al: Impaired endothelial progenitor cell activity is associated with reduced arterial elasticity in patients with essential hypertension. Clin Exp Hypertens 32: 444-452, 2010.

20. Yang Z, Wang JM, Chen L, Luo CF, Tang AL and Tao J: Acute exercise-induced nitric oxide production contributes to upregulation of circulating endothelial progenitor cells in healthy subjects. J Hum Hypertens 21: 452-460, 2007.

21. Vasa M, Fichtlscherer S, Aicher A, Adler K, Urbich C, Martin H, Zeiher AM and Dimmeler S: Number and migratory activity of circulating endothelial progenitor cells inversely correlate with risk factors for coronary artery disease. Circ Res 89: E1-E7, 2001.

22. Yang Z, Xia WH, Su C, Wu F, Zhang YY, Xu SY, Liu X, Zhang XY, Ou ZJ, Lai GH, et al: Regular exercise-induced increased number and activity of circulating endothelial progenitor cells attenuates age-related decline in arterial elasticity in healthy men. Int J Cardiol 165: 247-254, 2013.

23. Yang Z, Xia WH, Zhang YY, Xu SY, Liu X, Zhang XY, Yu BB, Qiu YX and Tao J: Shear stress-induced activation of Tie2-dependent signaling pathway enhances reendothelialization capacity of early endothelial progenitor cells. J Mol Cell Cardiol 52: 1155-1163, 2012.

24. Yang Z, Tao J, Wang JM, Tu C, Xu MG, Wang Y and Pan SR: Shear stress contributes to t-PA mRNA expression in human endothelial progenitor cells and nonthrombogenic potential of small diameter artificial vessels. Biochem Biophys Res Commun 342: 577-584, 2006.

25. Hirschi KK, Ingram DA and Yoder MC: Assessing identity, phenotype, and fate of endothelial progenitor cells. Arterioscler Thromb Vasc Biol 28: 1584-1595, 2008. 
26. Walther C, Gaede L, Adams V, Gelbrich G, Leichtle A, Erbs S, Sonnabend M, Fikenzer K, Korner A, Kiess W, et al: Effect of increased exercise in school children on physical fitness and endothelial progenitor cells: A prospective randomized trial. Circulation 120: 2251-2259, 2009.

27. Zeng W, Yuan W, Li L, Mi J, Xu S, Wen C, Zhou Z, Xiong J, Sun J, Ying D, et al: The promotion of endothelial progenitor cells recruitment by nerve growth factors in tissue-engineered blood vessels. Biomaterials 31: 1636-1645, 2010.

28. Heiss C, Keymel S, Niesler U, Ziemann J, Kelm M and Kalka C: Impaired progenitor cell activity in age-related endothelial dysfunction. J Am Coll Cardiol 45: 1441-1448, 2005.

29. Corretti MC, Anderson TJ, Benjamin EJ, Celermajer D, Charbonneau F, Creager MA, Deanfield J, Drexler H, Gerhard-Herman M, Herrington D, et al: Guidelines for the ultrasound assessment of endothelial-dependent flow-mediated vasodilation of the brachial artery: A report of the international brachial artery reactivity task force. J Am Coll Cardiol 39: 257-265, 2002.

30. Sibal L, Aldibbiat A, Agarwal SC, Mitchell G, Oates C, Razvi S, Weaver JU, Shaw JA and Home PD: Circulating endothelial progenitor cells, endothelial function, carotid intima-media thickness and circulating markers of endothelial dysfunction in people with type 1 diabetes without macrovascular disease or microalbuminuria. Diabetologia 52: 1464-1473, 2009.

31. Farooq V, Head SJ, Kappetein AP and Serruys PW: Widening clinical applications of the Syntax score. Heart 100: 276-287, 2014.

32. Chen SL, Chen JP, Mintz G, Xu B, Kan J, Ye F, Zhang J, Sun X, $\mathrm{Xu}$ Y, Jiang Q, et al: Comparison between the NERS (new risk stratification) score and the Syntax (synergy between percutaneous coronary intervention with taxus and cardiac surgery) score in outcome prediction for unprotected left main stenting. JACC Cardiovasc Interv 3: 632-641, 2010.
33. Yadav M, Palmerini T, Caixeta A, Madhavan MV, Sanidas E, Kirtane AJ, Stone GW and Généreux P: Prediction of coronary risk by Syntax and derived scores: Synergy between percutaneous coronary intervention with taxus and cardiac surgery. J Am Coll Cardiol 62: 1219-1230, 2013.

34. Cuenza L, Collado MP and Ho Khe Sui J: Global risk score and clinical Syntax score as predictors of clinical outcomes of patients undergoing unprotected left main percutaneous catheter intervention. Cardiol Res 8: 312-318, 2017.

35. Bentzon JF, Otsuka F, Virmani R and Falk E: Mechanisms of plaque formation and rupture. Circ Res 114: 1852-1866, 2014.

36. Flammer AJ, Anderson T, Celermajer DS, Creager MA, Deanfield J, Ganz P, Hamburg NM, Lüscher TF, Shechter M, Taddei S, et al: The assessment of endothelial function: From research into clinical practice. Circulation 126: 753-767, 2012.

37. Shyu KG: Enhancement of new vessel formation by angiopoietin-2/Tie2 signaling in endothelial progenitor cells: A new hope for future therapy? Cardiovasc Res 72: 359-360, 2006

38. Fulton D, Gratton JP, McCabe TJ, Fontana J, Fujio Y, Walsh K, Franke TF, Papapetropoulos A and Sessa WC: Regulation of endothelium-derived nitric oxide production by the protein kinase Akt. Nature 399: 597-601, 1999.

39. Thum T, Fraccarollo D, Schultheiss M, Froese S, Galuppo P Widder JD, Tsikas D, Ertl G and Bauersachs J: Endothelial nitric oxide synthase uncoupling impairs endothelial progenitor cell mobilization and function in diabetes. Diabetes 56: 666-674, 2007.

(i) (9) This work is licensed under a Creative Commons Attribution-NonCommercial-NoDerivatives 4.0 International (CC BY-NC-ND 4.0) License. 